\title{
El lado más sensible del esófago en relación con el reflujo gastroesofágico. Enseñanzas de ROMA IV
}

\author{
José Tawil, ${ }^{1}$ Ronnie Fass ${ }^{2}$ \\ ${ }^{1}$ Departamento de Esófago y Neurogastroenterología de Gedyt, Gastroenterología Diagnóstica y Terapéutica. Buenos Aires, Argentina. \\ ${ }^{2}$ Director del Departamento de Gastroenterología y Hepatología, jefe del Departamento de Esófago y Deglución, Metrohealth Medical Center. \\ Cleveland, EE.UU.
}

Acta Gastroenterol Latinoam 2020;50(1):72-81

Recibido: 18/07/2019 / Aceptado: 04/10/2019 / Publicado online: 23/03/2020 / https://doi.org/10.52787/pnzz8653

\section{Resumen}

Roma IV incorporó una nueva categoría diagnóstica dentro del grupo de los trastornos del esófago, denominada hipersensibilidad al reflujo. Este nuevo nombre reemplazó al término esófago hipersensible e incluye a pacientes con parámetros de reflujo normales pero con correlación sintomática con los eventos de reflujo gastroesofágico. En pacientes con pirosis sin respuesta a inhibidores de la bomba de protones es un diagnóstico a descartar junto con el de pirosis funcional, en la cual no existe correlación sintomática entre evento de reflujo y sintoma. La pirosis funcional, que denota falta de correlación entre sintomas y eventos de reflujo en el escenario de prueba de reflujo normal, y la hipersensibilidad al reflujo deben ser excluidas en pacientes que no responden al tratamiento con inhibidores de la bomba de protones. Los pacientes con hipersensibilidad al reflujo demuestran hipersensibilidad esofágica al reflujo (cualquier tipo) y tanto en aquellos con hipersensibilidad al reflujo como en los que tienen pirosis funcional, los sintomas son impulsados por sensibilización central y periférica. La correcta diferenciación permite un enfoque terapéutico con mayor chance de éxito. Los moduladores del dolor son el pilar del tratamiento de los pacientes con ambos trastornos, aunque aquellos con hipersensibilidad al reflujo pueden también mostrar respuesta al tratamiento antirreflujo.

Correspondencia: José Tawil

Correo electrónico: drjosetawil@gmail.com
Palabras claves. Reflujo gastroesofágico, hipersensibilidad al reflujo, pirosis funcional, desórdenes funcionales de esófago, desórdenes de la interacción cerebro-intestinal, Roma IV.

\section{The Most Sensitive Side of the Esophagus Related to Gastroesophageal Reflux. Teachings from Rome IV}

\section{Summary}

Rome IV incorporated a new diagnostic category into the functional esophageal disorders group called reflux hypersensitivity. This new name replaced the term hypersensitive esophagus and included patients with normal acid reflux parameters but with symptomatic correlation with reflux events. Functional heartburn, which denoted lack of correlation between symptoms and reflux events in the background of normal $p H$ test, and reflux hypersensitivity, should be excluded in heartburn patients who failed proton pump inhibitor treatment. Reflux hypersensitivity patients demonstrates esophageal hypersensitivity to reflux (any type), and both, reflux hypersensitivity and functional heartburn's symptoms are driven by central and peripheral sensitization. The correct differentiation between these two esophageal disorders allows a therapeutic approach with greater chance of success. Neuromodulators are considered the mainstay of treatment for these patients even though patients with reflux hypersensitivity demonstrate response to anti-reflux treatment as well.

Keywords. Gastro esophageal reflux, reflux hypersensitivity, functional heartburn, functional esophageal disorders, brain-gut interaction disorders, Rome IV. 


\section{Abreviaturas}

IBP: Inhibidores de la bomba de protones.

ERGE: Enfermedad por reflujo gastroesofágico.

EB: Esófago de Barrett.

ERNE: Enfermedad por reflujo no erosiva.

PF: Pirosis funcional.

HR: Hipersensibilidad al reflujo.

\section{Introducción}

La enfermedad por reflujo gastroesofágica (ERGE) se caracteriza clínicamente por pirosis y regurgitación. Si bien la prueba a la respuesta a los inhibidores de la bomba de protones (IBP) es hoy día el primer paso diagnóstico de la ERGE, es la endoscopía la que define el fenotipo en enfermedad por reflujo erosiva (ERE), esófago de Barrett (EB) y no erosiva (ERNE). Es este último grupo el que muestra la menor respuesta a los IBP y, en parte, esta menor respuesta se debe a que muchos de estos pacientes no tienen verdadera enfermedad por reflujo como causa de sus síntomas. La respuesta a los IBP dependerá de la relación causal entre el reflujo ácido y el síntoma. Sin embargo, en un porcentaje variable de pacientes, la pirosis puede ser generada por hipersensibilidad a un estímulo químico o mecánico que no siempre es ácido. La mejor categorización del paciente con pirosis permite mejorar la estrategia terapéutica y por ello es fundamental familiarizarse con los términos de hipersensibilidad al reflujo $(\mathrm{HR})$ y de pirosis funcional (PF) que, junto con el globus, la disfagia funcional y el dolor torácico esofágico funcional son los trastornos esofágicos dentro de la nueva clasificación de los trastornos de la interacción intestinocerebro de Roma IV.

\section{Como definen la hipersensibilidad al reflujo y la pirosis funcional y el porqué del cambio de criterios en ROMA IV}

Basado en el entendimiento de la fisiopatogenia de los síntomas de los pacientes con trastornos funcionales del tracto gastrointestinal, Roma IV modificó este término por el de trastornos de la interacción intestino-cerebro. En esta nueva clasificación de Roma IV, los trastornos esofágicos son el dolor torácico esofágico funcional, el globus, la disfagia funcional, la hipersensibilidad al reflujo y la pirosis funcional. ${ }^{1}$

El término pirosis funcional (PF) fue introducido a fines de la década de 1980 para categorizar a aquellos pacientes con síntomas de reflujo gastro-esofágico pero sin la esperada respuesta sintomática al tratamiento convencio- nal con inhibidores del ácido. En cambio, el término hipersensibilidad al reflujo es reciente, introducido en los criterios de Roma IV, ${ }^{1}$ para reemplazar al antes denominado esófago hipersensible dentro de los criterios de Roma II. ${ }^{2}$

En Roma II, el comité de expertos sugirió que los pacientes con pirosis y endoscopía alta normal se dividieran en aquellos con enfermedad por reflujo no erosiva (ERNE) $\mathrm{y}$ con pirosis funcional. ${ }^{2}$ El grupo de pirosis funcional incluía a pacientes con esófago hipersensible (aquellos cuyos síntomas se correlacionan con eventos de reflujo ácido) y a pacientes con pirosis no relacionada con eventos reflujo. En Roma III, en cambio, se dividió el grupo de ERNE en pacientes con exposición anormal al ácido esofágico, pacientes con esófago hipersensible y pacientes con síntomas no relacionados con el reflujo que respondían al tratamiento con inhibidores de la bomba de protones (IBP). ${ }^{3}$

Los criterios de trastornos funcionales esofágicos de Roma IV dividen a los pacientes con pirosis y endoscopía normal en tres grupos a partir del resultado del monitoreo de reflujo mediante $\mathrm{pH}$ impedanciometría realizado sin IBP. ${ }^{1}$ Estos tres grupos son los de aquellos pacientes con verdadera ERNE (exposición ácida anormal con o sin asociación sintomática), hipersensibilidad al reflujo (aquellos con exposición ácida normal, asociación sintomática positiva) y pirosis funcional (pacientes con exposición ácida normal y sin asociación sintomática). Roma IV considera que la hipersensibilidad al reflujo debe ser un trastorno en sí, por separado del resto, ${ }^{1}$ a diferencia de los criterios de Roma previos.

El principal motivo en la diferenciación de los tres grupos de ERNE radica en la conducta terapéutica, ya que en la verdadera ERNE el tratamiento estará especialmente dirigido a evitar los eventos de reflujo; en cambio, en los pacientes con HR el rol del ácido es menor y el de la sensorialidad y percepción es mayor, y en los pacientes con PF la supresión del ácido no juega ningún rol.

Si bien nunca dejó de cuestionarse la hipersensibilidad al reflujo, siempre se debatió si este grupo de pacientes tiene un trastorno esofágico funcional o una ERGE de baja intensidad, ya que todos los comités de Roma reconocieron las limitaciones de los índices de asociación de síntomas, pero al mismo tiempo entendieron que existe un subgrupo significativo de pacientes con pirosis que parecen desarrollar síntomas relacionados al reflujo en el rango fisiológico de la exposición ácida del esófago. ${ }^{1-3}$

Roma IV define la hipersensibilidad al reflujo como aquellos síntomas retro-esternales que incluyen pirosis y dolor torácico, presentes al menos dos veces por semana, durante los últimos tres meses, con inicio de los síntomas al menos seis meses previo al diagnóstico. Estos sínto- 
mas deben darse en ausencia de hallazgos endoscópicos, incluida la esofagitis eosinofílica, ausencia de trastornos motores esofágicos mayores y evidencia de gatillo de síntomas por eventos de reflujo pese a la exposición ácida normal en pHmetría o $\mathrm{pH}$ impedanciometría. ${ }^{1}$ Se acepta que la respuesta al IBP pueda ser parcial.

La pirosis funcional es definida por Roma IV como la sensación molesta o dolor quemante retro-esternal al menos dos veces por semana, que no mejora pese a dosis óptimas de inhibidores de bomba de protones y en ausencia de evidencia de reflujo gastroesofágico (exposición ácida anormal y asociación sintomática) y de trastornos motores mayores. Estos síntomas deben tener tres meses de duración y ser iniciados al menos seis meses previos al diagnóstico. ${ }^{1}$

Roma IV introdujo un nuevo concepto que no se mencionó en sus criterios anteriores, y es que los desórdenes funcionales del esófago, PF e HR, pueden solaparse con ERGE. Roma IV enfatizó que los pacientes con ERGE documentado que fallaron el tratamiento con IBP tienen probablemente una superposición con un trastorno esofágico funcional, que es probablemente el responsable de los síntomas de los pacientes. Este concepto es un progreso en nuestra comprensión de los mecanismos que conducen a los síntomas persistentes en pacientes con ERGE que están en tratamiento con IBP. También Roma IV vincula la hipersensibilidad esofágica y la comorbilidad psicológica como posibles mecanismos subyacentes para el fracaso del tratamiento con IBP en pacientes con ERGE. Un reciente estudio de Abdallah y col. ayudó a consolidar el concepto de superposición entre la ERGE y los trastornos esofágicos funcionales en pacientes con fracaso de IBP, al demostrar que el $75 \%$ de los pacientes con ERGE que continuaron con síntomas bajo una dosis de IBP diario tenía una superposición con pirosis funcional $(62,5 \%)$ o con hipersensibilidad al reflujo $(12,5 \%) .{ }^{4}$ Los autores concluyeron que la mayoría de los pacientes con ERGE que fallaron el tratamiento con IBP tienen una superposición con la pirosis funcional o hipersensibilidad al reflujo, que probablemente son responsables de la falta de respuesta al tratamiento.

\section{Prevalencia de HR y PF}

Siendo que uno de los principales motivos de las actualizaciones de los criterios de Roma es la búsqueda de la mayor sensibilidad y especificidad diagnóstica, la prevalencia ha sufrido modificaciones con los cambios en los criterios. Estos cambios tuvieron que ver con los avances de la endoscopía, la manometría y la $\mathrm{pH}$ impedanciometría, que permitieron comprender cada vez más la fisiopatogenia del heterogéneo grupo que conforman los pacientes con pirosis y endoscopía normal. ${ }^{5-6}$
Del total de pacientes con pirosis y endoscopía normal, aproximadamente el $40 \%$ tienen verdadera ERNE, el $35 \%$ HR y $25 \%$ PF. 5

Savarino y col. evaluaron con $\mathrm{pH}$ impedanciometría a 329 pacientes con pirosis y endoscopía normal y observaron que 36\% tenían hipersensibilidad al reflujo y $24 \%$ pirosis funcional. ${ }^{6}$ En un más reciente estudio de 351 pacientes con pirosis y endoscopía normal en los que se realizó $\mathrm{pH}$ impedanciometría, $41 \%$ de pacientes tuvieron pirosis funcional y $25,9 \%$, hipersensibilidad al reflujo. $^{7}$

En cambio, la prevalencias de estos trastornos funcionales esofágicos en pacientes refractarios a doble dosis de IBP es variable. En un estudio en pacientes con síntomas de reflujo pese a doble dosis de IBP, la prevalencia de pirosis funcional fue de alrededor del $54 \%,{ }^{8}$ y en otro fue del $55 \% .{ }^{9}$ Savarino y col. evaluaron a 219 pacientes tratados y observaron 39\% de pirosis funcional y $28 \%$ de hipersensibilidad al reflujo. ${ }^{10}$ En otro estudio, Patel y col. observaron $29 \%$ de hipersensibilidad al reflujo y que, de estos, el 6,5\% era sensible al reflujo ácido, 50,6\% al débilmente ácido y $42,8 \%$ a ambos. ${ }^{11}$ Ribolsi y col. analizaron a 92 pacientes refractarios a IBP y estudiados con endoscopía alta y $\mathrm{pH}$ impedanciometría y observaron que $42 \%$ tenían hipersensibilidad al reflujo y $26 \%$ pirosis funcional. ${ }^{12}$

Así parece que en pacientes con pirosis y endoscopía normal que no mejoran con doble dosis de IBP, el porcentaje de pacientes con pirosis funcional e hipersensibilidad al reflujo es cercano al $90 \% .^{13}$

\section{Características clínicas}

La falta de respuesta a una terapia de supresión ácida agresiva definida como una doble dosis de IBP debe hacer sospechar, entre otras causas, la posibilidad de pirosis funcional o de HR.

Las características clínicas de ambos trastornos esofágicos serían las mismas en cuanto a severidad, frecuencia o duración de la pirosis. ${ }^{14}$ Shapiro y col. mostraron que en el género femenino $(66,5 \%)$, la presencia de síndrome de intestino irritable $(48,2 \%)$ y ansiedad $(35,8 \%)$ se asociaban con hipersensibilidad al reflujo, al igual que la presencia de depresión (6\%) y consumo de alcohol (39\%). Este mismo grupo demostró que, comparados con pacientes con ERNE, los pacientes con pirosis funcional tienen el mismo predominio femenino y edad promedio de 46 años. ${ }^{15}$ Ambos trastornos están asociados con otros trastornos del tracto gastrointestinal, como dolor torácico, dispepsia y síndrome de intestino irritable. ${ }^{16}$ Los pacientes con PF son predominantemente mujeres, con larga historia de pirosis, y mayor comorbilidad psicológica ${ }^{17}$ (Tabla 1). 
Tabla 1. Diferencia clínica entre hipersensibilidad al reflujo y pirosis funcional

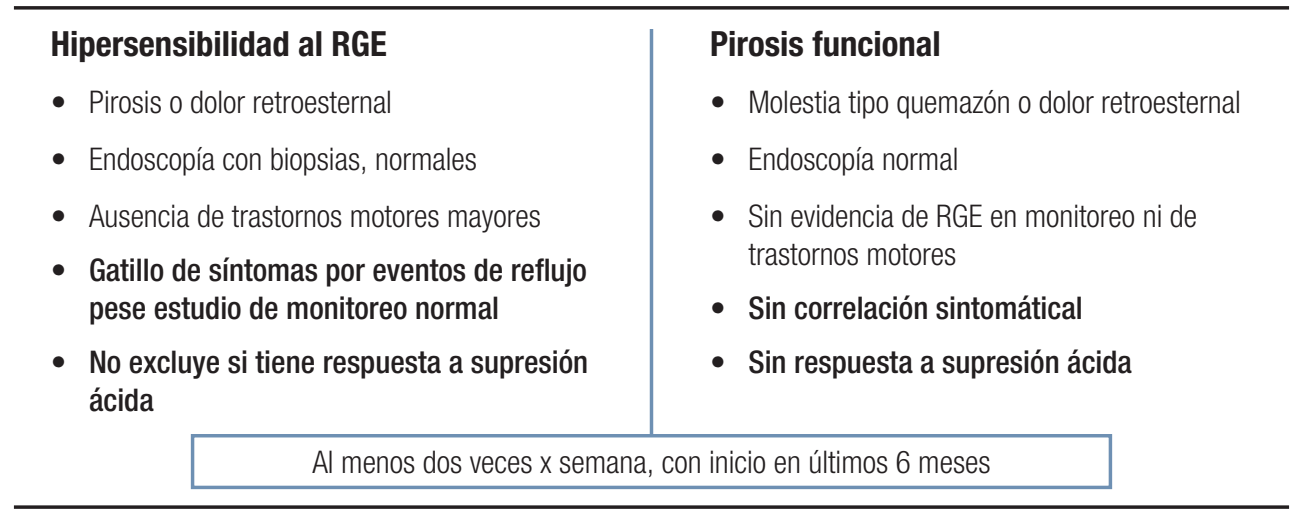

\section{¿Cuál es la patofisiología en estos trastornos esofágicos?}

Como en otros trastornos funcionales gastrointestinales, denominados trastornos de la interacción intestinocerebro, desde Roma IV, la hipersensibilidad visceral desempeña un papel importante en la generación de síntomas en pacientes con HR y PF. La hipersensibilidad visceral se define como la percepción de estímulos no dolorosos como dolorosos (alodinia) y estímulos dolorosos como más dolorosos (hiperalgesia).

Algunos mecanismos neuronales centrales, como las comorbilidades psicológicas (ansiedad y depresión), la privación del sueño, estrés o hipervigilancia, pueden modular la percepción esofágica y hacer que los pacientes perciban estímulos esofágicos de baja intensidad como dolorosos. Los estudios que utilizan potenciales evocados corticales o RMN funcional durante la distensión con globo del esófago o la perfusión ácida han demostrado que el procesamiento central de las señales del esófago varía entre los controles normales y los pacientes con ERGE, HR o PF. ${ }^{18}$

La HR se caracteriza por la hipersensibilidad con cantidades fisiológicas de reflujo gastroesofágico, tanto ácido como no ácido.

Los pacientes con HR muestran más alteraciones en el funcionamiento esofágico en comparación con aquellos con PF. Por ejemplo, la prevalencia de esofagitis microscópica, un marcador potencial de reducción de integridad de la mucosa, fue del $65 \%$ en pacientes con HR, comparado con $13 \%$ en pacientes con PF y $15 \%$ en voluntarios sanos. En cambio, la prevalencia de esofagitis microscópica en pacientes con PF resultó similar a la de voluntarios sanos. ${ }^{19}$ En otro estudio, la integridad de la mucosa esofágica y la tasa de aclaramiento químico esofágico estaban reducidos en pacientes con HR comparado con pacientes con PF y voluntarios sanos. ${ }^{20}$ Así como la esofagitis microscópica es más prevalente y la integridad de la mucosa esofágica y el vaciamiento esofágico están más alterados en pacientes con ERGE comparado con los pacientes con HR, y en estos comparado con aquellos con $\mathrm{PF}$, los pacientes con HR experimentan pirosis a pesar de una mayor protección mucosa de los receptores esofágicos al estímulo químico, invocando mecanismos de mayor sensibilidad de los receptores. ${ }^{21}$ La HR puede involucrar alteración de la función de barrera mucosa esofágica, permitiendo una mayor exposición de los receptores periféricos a los distintos químicos del reflujo, sensibilización de estos receptores y también sensibilización central, la que puede ocurrir tras activaciones repetidas de los receptores periféricos, o generados a nivel central por estrés, en ambos casos incrementando la percepción del estímulo. ${ }^{21}$

En otro estudio se observó que los pacientes con HR muestran un mayor grado de eventos de reflujo proximales y eventos de reflujo débilmente ácidos comparados con pacientes con $\mathrm{PF}$ y voluntarios sanos. ${ }^{5}$

En pacientes con PF, el síntoma ocurre en presencia de niveles fisiológicos de reflujo, donde el mecanismo de generación del síntoma está más relacionado con el incremento de la percepción y no con el incremento del estímulo. Así, un paciente puede desarrollar pirosis funcional tras la activación repetitiva pero no actual de receptores periféricos por eventos de reflujo. Los pacientes con PF no muestran cambios en la mucosa en estudios con impedancia de mucosa ni histológicos. Más que un daño directo, parecería estar involucrada una inflamación de bajo grado con efectos neuroinmunológicos como principal mecanismo de sensibilización periférica. ${ }^{22}$ Los pacientes con PF son más sensibles a la distensión con balón esofágico, comparados con voluntarios sanos. ${ }^{18}$ La alodinia es la inapropiada respuesta al dolor a un estímulo que se encuentra dentro del rango fisiológico normal. Debido a que este es un mecanismo más relacionado con la sensibilización central que periférica podría ser un mecanismo contribuyente a la pirosis en pacientes con PF. ${ }^{23}$ Esta 
hipersensibilidad central puede ser gatillada por exposición previa al ácido, otras causas de inflamación leve o por factores emocionales, contribuyendo a la PF. Así, los factores psicológicos pueden incrementar la percepción del estímulo, y la presencia de comorbilidad psiquiátrica es elevada en pacientes con PF. ${ }^{24}$

En pacientes con ERGE que se encuentran bajo tratamiento con IBP, el reflujo pudo haber sido resuelto pero no necesariamente así la sensibilización central o periférica. Este concepto puede traer dificultades en su comprensión, pero es muy importante para entender la falta de respuesta sintomática a los IBP en pacientes con historia previa de ERGE. En este caso, la exposición ácida no es actual y por ello la posibilidad de respuesta al IBP es baja.

La distensión esofágica causada por un importante volumen refluido puede incrementar la sensibilidad de los receptores esofágicos periféricos al ácido. Así, la sensibilidad a la distensión está aumentada en pacientes con ERGE tras la perfusión esofágica con ácido ${ }^{25}$ y la percepción del síntoma está aumentada en pacientes con ERNE cuando en el reflujo está presente gas. ${ }^{26}$

\section{¿Cómo llegar al diagnóstico de HR y PF?}

De acuerdo con los criterios de Roma IV para los trastornos esofágicos, la exposición ácida y no ácida y la asociación sintomática durante la $\mathrm{pH}$-impedanciometría son fundamentales para el diagnóstico de HR (exposición esofágica ácida total normal pero correlación sintomática con los eventos de reflujo ácido o no ácido) y PF (exposición ácida normal, sin correlación sintomática).

Siendo que la primera medida ante un paciente con síntomas de reflujo es la prueba a la respuesta a inhibidores de la bomba de protones, los estudios diagnósticos como la endoscopía, la manometría esofágica y la $\mathrm{pH}$ impedanciometría se reservan para aquellos pacientes que no responden a la misma luego de dos meses. ${ }^{27}$

La endoscopía digestiva alta en pacientes con pirosis que no responden a IBP sirve para confirmar el diagnóstico de enfermedad por reflujo en caso de hallar esofagitis y esófago de Barrett. Lo cierto es que el porcentaje de pacientes con esofagitis bajo tratamiento con IBP es bajo. ${ }^{28}$ En ausencia de signos de ERGE es necesario tomar biopsias para descartar signos histológicos de esofagitis ${ }^{29} \mathrm{y}$ esofagitis eosinofílica (EoE). Si bien la disfagia es el principal síntoma de la EoE, hasta el $30 \%$ de los pacientes reportan pirosis. ${ }^{30}$ Las biopsias de esófago en pacientes con pirosis sin respuesta a los IBP incrementan el rendimiento en el diagnostico de la EoE hasta en un $4 \% \cdot{ }^{31}$ En estudios recientes, Ates y col. reportaron que la medición de la impedancia de la mucosa esofágica a través de un ca- téter podría diferenciar pacientes con ERGE de aquellos con pirosis funcional con mayores niveles de especificidad y valores predictivos que el monitoreo inalámbrico. ${ }^{32}$ Al mismo tiempo, la endoscopía permite descartar otras causas menos frecuentes de síntomas de reflujo, como acalasia y lesiones orgánicas.

Sin duda el método diagnóstico más importante en la evaluación del paciente con pirosis sin respuesta al tratamiento farmacológico es el monitoreo de reflujo gastroesofágico, cuyo fin es establecer o descartar si el reflujo es el causante de la pirosis y distinguir entre ERGE, HS y PF. La pH-impedanciometría de 24 horas es hoy el método de oro en el diagnóstico de la enfermedad por reflujo $\mathrm{y}$ es fundamental para la categorización del paciente con pirosis sin respuesta a la supresión ácida. ${ }^{33} \mathrm{La} \mathrm{pH}$ impedanciometría tiene ventajas sobre la pHmetría convencional. Al menos dos estudios demostraron que el 37-57\% de los pacientes con síntomas persistentes bajo IBP correlacionan con episodios de reflujo no ácidos, y detectaron más pacientes con síntomas relacionados con reflujo que la pHmetría convencional. ${ }^{5-34}$ En los últimos años se han agregado algunos parámetros que han colaborado con la detección de reflujo, como la medición de la impedancia basal, que refleja el daño de la integridad de la mucosa aun en ausencia de daño macroscópico. ${ }^{35} \mathrm{El}$ otro parámetro es el índice de la onda peristáltica de deglución inducida post reflujo. La combinación de estos dos nuevos parámetros logra incrementar la sensibilidad en la definición de los pacientes con enfermedad por reflujo no erosiva tanto sin o con IBP. ${ }^{35}$ Los pacientes con HR muestran valores de índice de la onda peristáltica de deglución inducida post reflujo y de impedancia basal nocturna promedio significativamente menores comparado con voluntarios sanos y pacientes con pirosis funcional. ${ }^{5}$

La $\mathrm{pH}$ impedanciometría, como cualquier método en el cual la interpretación de los síntomas es fundamental para un diagnóstico certero, requiere la participación del paciente en el registro de cada evento sintomático. La correlación sintomática en un paciente con monitoreo ácido esofágico normal es decisiva para diferenciar HR de PF.

Uno de los métodos reconocidos como estadísticamente más sencillo es el SAP (Symptom Association Probability) o PAS (Probabilidad de Asociación Sintomática), sin embargo, ha demostrado no ser óptimo para predecir la respuesta al tratamiento (valor predictivo positivo $79 \%$, sensibilidad $73 \%$ y especificidad $65 \%$ ), ${ }^{36}$ siendo la probabilidad de la positividad del PAS muy baja, de 4-33\% (mejora en la medida en que el número de episodios de reflujo se incrementa) y muestra una variabilidad día a día en pacientes que se realizan el estudio sin IBP. ${ }^{37}$ Otro de los principales inconvenientes es la dificultad que 
tienen los pacientes en marcar cada evento sintomático. ${ }^{38}$ Choksi y col. confirmaron la variabilidad diaria, siendo los pacientes PAS positivo un día y negativo al otro, así como también observaron que las diferencias en la correlación síntoma-reflujo que crean la positividad del PAS son pequeñas, dejando la duda acerca del rol del PAS en el diagnóstico diferencial de la HR y la PF. ${ }^{39}$

Para poder contrarrestar la variabilidad diaria que ocurre aproximadamente en el $25 \%$ de los pacientes, ${ }^{40}$ se puede evaluar la exposición ácida esofágica y su asociación sintomática mediante la colocación vía endoscópica de la cápsula inalámbrica, que permite registros de hasta 96 horas y se puede realizar en pacientes sin tratamiento y bajo tratamiento. Este es un método de medición de reflujo ideal para pacientes con síntomas de reflujo típicos o atípicos sin respuesta a IBP sin evidencia de reflujo en la endoscopía, en los cuales se intenta descartar la en- fermedad por reflujo como responsable de los síntomas, aunque no permite la detección de eventos de reflujo débilmente ácidos o no ácidos. ${ }^{40}$

La manometría esofágica tiene la función de descartar trastornos motores esofágicos mayores como causa de los síntomas refractarios y se realiza en el instante previo a la $\mathrm{pH}$ o $\mathrm{pH}$ impedanciometría de 24 horas ya que además permite localizar el esfínter esofágico inferior. En el caso de haber monitoreado el reflujo con cápsula inalámbrica, puede realizarse luego de esta ante la ausencia de hallazgo de reflujo. Gao y col. compararon los hallazgos de la manometría en 36 voluntarios sanos, 147 pacientes con DF y 91 pacientes con HR y observaron que los pacientes con HR muestran mayor porcentaje de motilidad esofágica inefectiva y de peristalsis fragmentada. ${ }^{7}$ En la Figura 1 se propone un algoritmo diagnóstico.

Figura 1. Algoritmo diagnóstico propuesto

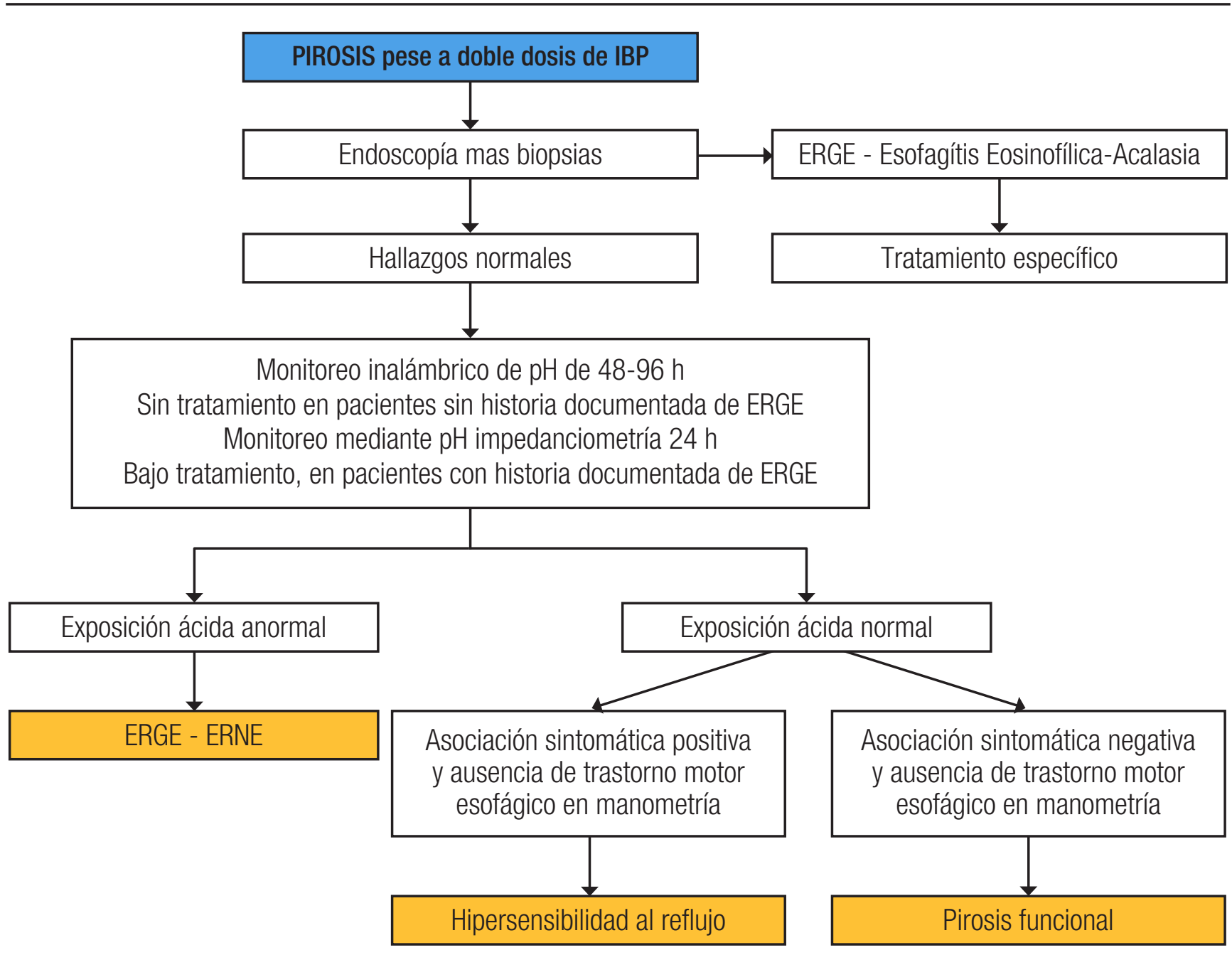

IBP: inhibidor de la bomba de protones; ERGE: enfermedad por reflujo gastroesofágica; ERNE: enfermedad por reflujo no erosiva; pH: pHmetría. 


\section{Tratamiento de la HR y PF}

\section{Cambios en estilo de vida}

Siendo que los síntomas del paciente con hipersensibilidad al reflujo son desencadenados por un evento de reflujo, todas las medidas de cambios de hábito de vida que impactan sobre la ERGE deben ser implementadas. Según estudios recientes, el descenso de peso y dejar de fumar deben ser recomendados al igual que evitar las comidas tarde en la noche y elevar la cabecera de la cama en pacientes con síntomas nocturnos. ${ }^{41}$

\section{Tratamiento farmacológico}

a) Antirreflujo: en los pacientes con hipersensibilidad al reflujo, los IBP, tanto en dosis estándar como en doble dosis están indicados, ya que minimizan la exposición ácida esofágica, pero no queda en claro aún cuál es la dosis máxima para este grupo de pacientes. En pacientes aún refractarios a doble dosis de IBP, el agregado de baclofeno (reduce las relajaciones transitorias del esfínter esofágico inferior), proquinéticos (incrementan el aclaramiento esofágico) y alginatos (especialmente el alginato de sodio) pueden ser beneficiosos en aquellos pacientes que aún demuestran presencia de $\mathrm{HR}$ al reflujo ácido o no ácido. El uso de ranitidina ha demostrado disminuir la secreción ácida y, a diferencia de los IBP, también ha demostrado reducir la sensibilidad esofágica al ácido, ${ }^{42}$ por lo que los pacientes con HR podrían beneficiarse y de hecho podría sumarse al tratamiento con IBP, aunque algunos autores han observado una disminución de su efectividad a largo plazo. Siendo los pacientes con HR un grupo de pacientes en los cuales la fisiopatogenia va más allá de la presencia de material refluido en esófago, el tratamiento endoscópico o quirúrgico es solo reservado para un limitado número de pacientes. ${ }^{43}$

b) Neuromodulación: Bilgi y col. evaluaron las comorbilidades psiquiátricas en pacientes con ERGE y observaron que aquellos con HR mostraron mayor ansiedad generalizada y que aquellos con PF tenían más depresión. ${ }^{44}$

Esta comorbilidad psicológica (ansiedad, estrés, depresión) puede modular la percepción esofágica y favorecer tanto la percepción de estímulos esofágicos de baja intensidad como de estímulos dolorosos. ${ }^{45}$

Debido a esta comorbilidad asociada a la hipersensibilidad visceral de estos trastornos funcionales, tanto en pacientes con pirosis funcional como en aquellos con hipersensibilidad al reflujo, se propone la utilización de neuromoduladores. El mecanismo de acción de estos incluye un efecto antidepresivo y también una acción anal- gésica que mejora la percepción del dolor visceral a través del eje sistema nervioso central-intestino. ${ }^{46}$

Dentro de los neuromoduladores se encuentran los antidepresivos tricíclicos (amitriptilna, imipramina), los inhibidores de la recaptación de serotonina (sertralina, paroxetina, citalopram, fluoxetina y trazodone) y los inhibidores de la recaptación de serotonina y noradrenalina, como la venlafaxina. Los estudios de estas drogas en patología funcional esofágica son pocos, incluyen a pocos pacientes y muchos de ellos tienen limitaciones. ${ }^{46}$

Los antidepresivos tricíclicos han mostrado ser eficaces en el tratamiento de trastornos funcionales esofágicos como el dolor torácico funcional, el dolor torácico no cardiogénico y el globus faríngeo. Debido a sus efectos anticolinérgicos (palpitaciones, sed) y sedativos, su dosis inicial es baja, de 5-10mg, y suelen indicarse de noche.

Los inhibidores de la recaptación de la serotonina han sido utilizados en dolor torácico funcional, pirosis funcional, dolor torácico no cardiogénico e hipersensibilidad al reflujo. ${ }^{47}$ Presentan menos efectos adversos que los tricíclicos.

En síntesis, en pacientes con HR es recomendable el agregado de neuromoduladores a la supresión ácida y en los pacientes con PF su utilización como primera opción, al mismo tiempo que suspender la supresión ácida.

\section{Tratamiento no farmacológico}

Las intervenciones psicológicas, la medicina alternativa y complementaria, así como otros enfoques no tradicionales pueden tener un papel como única terapia o parte de un enfoque terapéutico orientado a la hipersensibilidad al reflujo. Sin embargo, actualmente todavía estamos desprovistos de estudios que evalúen su valor terapéutico en esta población de pacientes.

En cambio, las intervenciones psicológicas se han evaluado en pacientes con pirosis funcional. En un estudio prospectivo, los autores demostraron que la biorretroalimentación era eficaz en dolor torácico no cardiogénico pero no en pirosis funcional. ${ }^{48}$ Por el contrario, la hipnoterapia se ha establecido como una intervención preferida para la pirosis funcional. En un estudio, los autores demostraron que había cambios constantes y significativos en los síntomas de la pirosis, la hipervigilancia esofágica, la calidad de vida y una tendencia a la mejora en la sensación de catástrofe en pacientes con pirosis funcional que recibieron siete semanas de hipnoterapia. ${ }^{49}$

El papel de la medicina alternativa y complementaria en la pirosis funcional ha sido apenas estudiado; se ha demostrado que la acupuntura mejora la pirosis diurna y nocturna como un agregado a la terapia en 
pacientes con pirosis que han fallado al tratamiento con dosis estándar de IBP versus duplicar la dosis de IBP..$^{50}$ Sin embargo, el grupo de pacientes estudiado no fue bien caracterizado, pero es probable que la pirosis funcional sola o en superposición con ERGE representara la mayoría de estos pacientes.
En la Tabla 2 se resumen las alternativas terapéuticas para HR y PF.

En la Figura 2 se propone un algoritmo diagnóstico y terapéutico para pacientes con pirosis sin respuesta al tratamiento con inhibidores de la bomba de protones según los distintos diagnósticos a partir del monitoreo de reflujo.

Tabla 2. Comparación de las opciones terapéuticas para $H R$ y PF

\begin{tabular}{|c|c|c|c|}
\hline Opciones & HR & PF & \multirow{13}{*}{$\begin{array}{l}\text { IBP: inhibidores de la bomba de protones. } \\
\text { IRS: inhibidores de la recaptación de serotonina. } \\
\text { ADTC: antidepresivos tricíclicos. } \\
\text { IRSyN: inhibidores recaptación serotonina y noradrenalina. }\end{array}$} \\
\hline IBP & $\checkmark$ & $\odot$ & \\
\hline Bloqueantes receptores $\mathrm{H}_{2}$ & $\checkmark$ & $\checkmark$ & \\
\hline Baclofeno & $\checkmark$ & $\odot$ & \\
\hline Alginatos & $\checkmark$ & $\ominus$ & \\
\hline Procinéticos & $\checkmark$ & $\odot$ & \\
\hline Tratamiento endoscópico & $\checkmark$ & $\odot$ & \\
\hline Cirugía & $\checkmark$ & 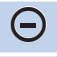 & \\
\hline IRS & $\checkmark$ & $\checkmark$ & \\
\hline ADTC & $\checkmark$ & $\checkmark$ & \\
\hline IRSyN & $\checkmark$ & $\checkmark$ & \\
\hline Intervenciones psicológicas & $\checkmark$ & $\checkmark$ & \\
\hline Hipnoterapia & $\checkmark$ & $\checkmark$ & \\
\hline
\end{tabular}

Figura 2. Algoritmo diagnóstico y terapéutico en paciente con pirosis sin respuesta a IBP

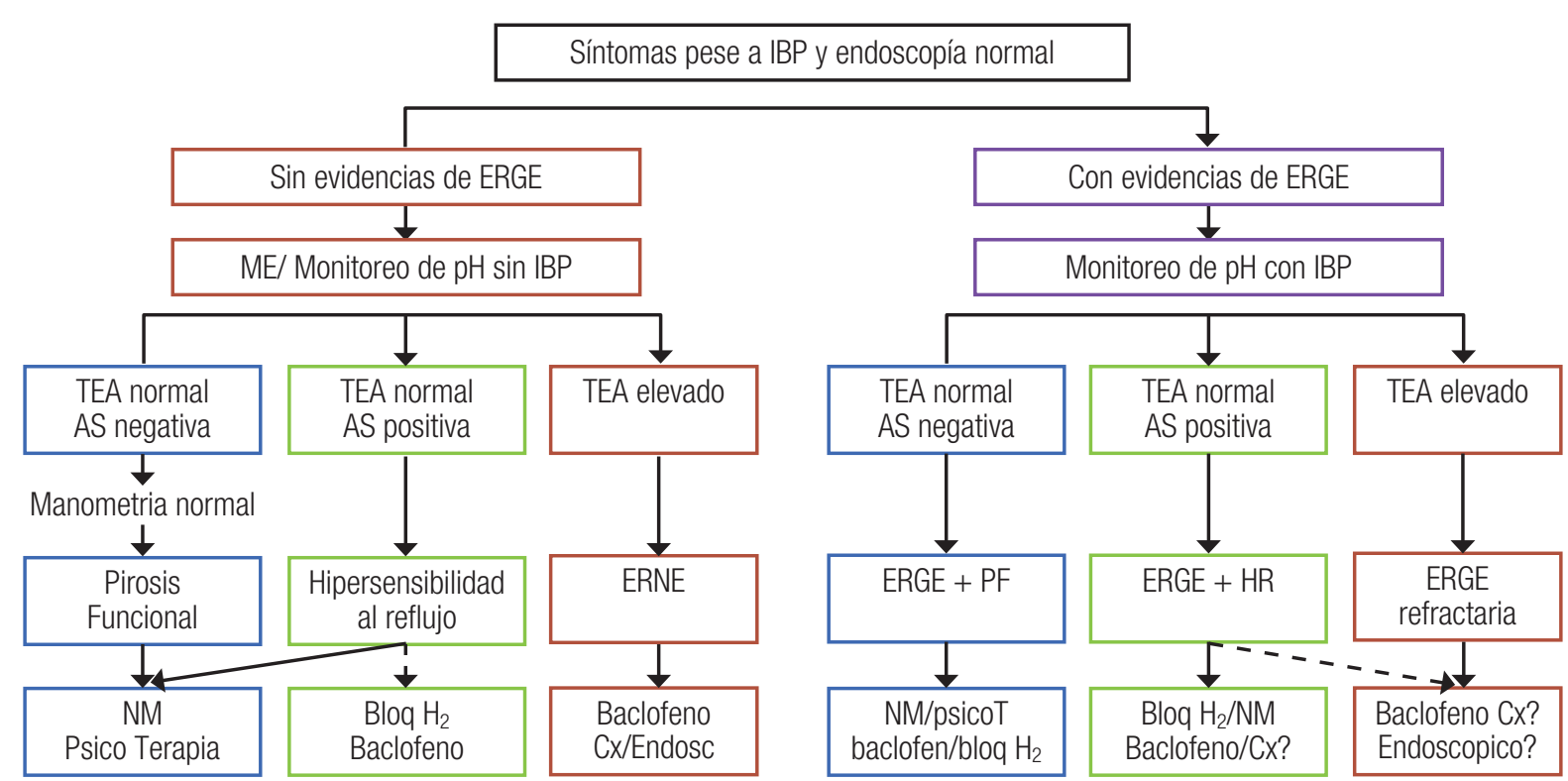

IBP: inhibidor bomba protón; ERGE: enfermedad reflujo gastroesofágica; ME: manometría esofágica; pH: monitoreo reflujo; TEA: tiempo exposición ácido; AS: asociación sintomática; ERNE: enfermedad reflujo no erosiva; PF: pirosis funcional; HR: hipersensibilidad al reflujo; NM: neuromodulación; $\mathrm{H}_{2}$ : histamina 2; Cx: cirugía. 


\section{Conclusiones}

En aquellos pacientes con pirosis sin respuesta al tratamiento convencional es fundamental descartar otros diagnósticos probables, y dentro de ellos, trastornos de la interacción intestino-cerebro como la pirosis funcional y la hipersensibilidad al reflujo, definidos a partir de Roma IV. El correcto abordaje permite hacer el diagnóstico más aproximado hacia cuál de los mecanismos fisiopatológicos es el responsable de la causa del síntoma y así realizar el tratamiento más apropiado.

\section{Aviso de derechos de autor}

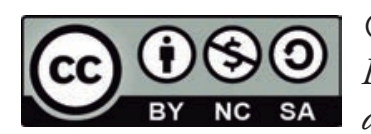

(C) 2021 Acta Gastroenterológica Latinoamericana. Este es un artículo de acceso abierto publicado bajo los términos de la Licencia Creative Commons Attribution (CC BY-NC-SA 4.0), la cual permite el uso, la distribución $y$ la reproducción de forma no comercial, siempre que se cite al autor y la fuente original.

Cite este artículo como: Tawil J, Fass R. El lado más sensible del esófago en relación con el reflujo gastroesofágico. Enseñanzas de ROMA IV. Acta Gastroenterol Latinoam. 2020;50(1):72-81. https://doi.org/10.52787/pnzz8653

\section{Referencias}

1. Aziz Q, Fass R, Gyawali CP, Miwa H, Pandolfino JE, Zerbib F. Esophageal disorders. Gastroenterology. 2016; 150(6): 1368-1379.

2. Clouse RE, Richter JE, Heading RC, Janssens J, Wilson JA. Functional esophageal disorders. Gut 1999; 45 (Suppl II): II31-II36.

3. Drossman DA. Editor. Rome III: The Functional Gastrointestinal Disorders. $3^{\text {rd }}$ ed. McLean (VA): Degnon Associates; 2006.

4. Abdallah J, George N, Yamasaki T, Ganocy S, Fass R. Most Patients with Gastroesophageal Reflux Disease Who Failed Proton Pump Inhibitor Therapy also have Functional Esophageal Disorders. Clin Gastroenterol Hepatol. 2018 Jun 18. [Epub ahead of print]

5. Savarino E, Zentilin P, Savarino V. NERD: an umbrella term including heterogeneous subpopulations. Nat Rev Gastroenterol Hepatol 2013; 10: 371-380.

6. Savarino E, Zentilin P, Tutuian R, Pohl D, Gemignani L, Malesci A, Savarino V. Impedance-pH reflux patterns can differentiate non-erosive reflux disease from functional heartburn patients. J Gastroenterol. 2012; 47(2): 159-168.

7. Gao F, Gao Y, Chen X, Zhang J. Comparison of esophageal function tests in chinese patients with functional hertburn and reflux hypersensitivity. Gastroenterol Res Pract 2017; 2017: 3596148.

8. Mainie I, Tutuian R, Shay S, Vela M, Zhang X, Sifrim D, Castell DO. Acid and non-acid reflux in patients with persistent symptoms despite acid suppressive therapy: a multicentre study using combined ambulatory impedance-pH monitoring. Gut 2006; 55 (10): 1398-1402.
9. Roman S, Keefer L, Imam H, Korrapati P, Mogni B Eident K, Friesen L, Kahrilas PJ, Martinovic Z, Pandolfino JE. Majority of symptoms in esophageal reflux PPI non-responders are not related to reflux. Neurogastroenterol Motil 2015; 27 (11): 1667-1674.

10. Savarino E, Marabotto E, Zentilin P, Frazzoni M, Sammito G, Bonfanti D, Sconfienza L, Assandri L, Gemignani L, Malesci A, Savarino $\mathrm{V}$. The added value of impedance-pH monitoring to Rome III criteria in distinguishing functional heartburn from non-erosive reflux disease. Dig Liver Dis 2011; 43: 542-547.

11. Patel A, Sayuk GS, Gyawali CP. Prevalence, characteristics, and treatment outcomes of reflux hypersensitivity detected on $\mathrm{pH}$ impedance monitoring. Neurogastroenterol Motil 2016; 28: 1382-1390.

12. Ribolsi M, Cicala M, Zentilin P, Efthymakis K, Petitti T, Mauro A, Savarino V, Penagini R. Prevalence and clinical characteristics of refractoriness to optimal protom pump inhibitor therapy in non-erosive reflux disease. Aliment Pharmacol Ther 2018; 48 (10): 1074-1081.

13. Yamasaki T, Fass R. Reflux hypersensitivity: a new functional esophageal disorder. J Neurogastroenterol Motil 2017; 23: 495-503.

14. Park EY, Choi MG, Baeg M, Lim CH, Kim J, Cho Y, Park J, Lee I, Kim S, Choi K. The value of early wireless esophageal $\mathrm{pH}$ monitoring in diagnosing functional heartburn in refractory gastroesophageal reflux disease. Dig Dis Sci 2013; 58(10): 2933-2939.

15. Shapiro M, Green C, Bautista JM, Peru RL, Malagon IB, Corvo M, Risner-Adler S, Tuchinsky I, Fass R. Functional heartburn patients demonstrate traits of functional bowel disorder but lack a uniform increase of chemoreceptor sensitivity to acid. Am J Gastroenterol 2006; 101 (5): 1084-1091.

16. de Bortoli N, Frazzoni L, Savarino EV, Frazzoni M, Martinucci I, Jania A, Tolone S, Scagliarini M, Bellini M, Marabotto E, Furnari M, Bodini G, Russo S, Bertani L, Natali V, Fuccio L, Savarino V, Blandizzi C, Marchi S. Functional heartburn overlaps with irritable bowel syndrome more often than GERD. Am J Gastroenterol 2016; 111: 1711-1717.

17. Yamasaki T, O’Neil J, Fass R. Update on functional heartburn. Gastroenterol Hepatol 2017; 13 (12): 725-734.

18. Yang M, Li ZS, Chen DF, Zou W, Xu XR, Fang DC, Xu GM, Stephens RL, Wang ZG. Quantitative assessment and characterization of visceral hyperalgesia evoked by esophageal balloon distention and acid perfusion in patients with functional heartburn, nonerosive reflux disease, and erosive esophagitis. Clin J Pain 2010; 26: 326-331.

19. Savarino E, Zentilin P, Mastracci L, Dilbecco P, Marabotto E, Gemignani L, Bruzzone L, de Bortoli N, Frigo AC, Fiocca R, Savarino $\mathrm{V}$. Microscopic esophagitis distinguishes patients with non- erosive reflux disease from those with functional heartburn. J Gastroenterol 2013; 48: 473-482.

20. Frazzoni M, de Bortoli N, Frazzoni L, Fumari M, Martinucci I, Tolone S, Farioli A, Marchi S, Fuccio L, Savarino V, Savarino E. Impairment of chemical clearance and mucosal integrity distinguishes hypersensitive esophagus from functional heartburn. J Gastroenterol 2017; 52: 444-451.

21. Hungin A, Molloy-Bland M, Scarpignato C. Revisiting Montreal: New insights into symptoms and their causes, and implications for the future of GERD. Am J Gastroenterol 2018 Oct 15. [Epub ahead of print]

22. Souza RF, Huo X, Mittal V, Schuler CM, Carmack SW, Zhang HI, Zhang X, Yu C, Hormi-Carver K, Genta RM, Spechler SM. Gastroesophageal reflux might cause esophagitis through a cytokine-mediated mechanism rather than caustic acid injury. Gastroenterology 2009; 137: 1776-1784. 
23. Miwa H, Kondo T, Oshima T, Fukuli H, Tomita T, Watari J. Esophageal sensation and esophageal hypersensitivity - overview from bench to bedside. J Neurogastroenterol Motil 2010; 16: 353-362.

24. Fass R, Naliboff BD, Fass SS, Peleg N, Wendel C, Malagon IB, Mayer EA. The effect of auditory stress on perception of intraesophageal acid in patients with gastroesophageal reflux disease. Gastroenterology 2008; 134: 696-705.

25. Lee KJ, Kwon HC, Cheong JY, Cho SW. Demographic, clinical, and psychological characteristics of the heartburn groups classified using the Rome III criteria and factors associated with the responsiveness to proton pump inhibitors in the gastroesophageal reflux disease group. Digestion 2009; 79: 131-136.

26. Emerenziani S, Sifrim D, Habib FI, Ribolsi M, Guarino MP, Rizzi M, Caviglia R, Pattiti T, Cicala M. Presence of gas in the refluxate enhances reflux perception in non-erosive patients with physiological acid exposure of the oesophagus. Gut 2008; 57 : 443-447.

27. Katz PO, Gerson LB, Vela MF. Guidelines for the diagnosis and management of gastroesophageal reflux disease. Am J Gastroenterol 2013.

28. Shaheen NJ, Denison H, Bjorck K, Silberg DG. Esophageal mucosal breaks in gastroesophageal reflux disease partially responsive to proton pump inhibitor therapy. Am J Gastroenterol 2013; 108 (4): 529-534.

29. Vela MF, Craft BM, Sharma N, Freeman J, Hazen-Martin D. Refractory heartburn: comparison of intercellular space diameter in documented GERD vs. functional heartburn. Am J Gasroenterol 2011; 106 (5): 844-850.

30. Remedios M, Campbell C, Jones DM, Kerlin P. Eosinophilic esophagitis in adults: clinical, endoscopic, histologic findings, and response to treatment with fluticasone propionate. Gastrointest Endosc 2006; 63: 3-12.

31. García-Compean D, González González JA, Marrufo García CA, Flores Gutiérrez JP, Barboza Quintana O, Galindo Rodríguez G, Mar Ruiz MA, De León Valdez D, Jaquez Quintana JO, Maldonado Garza HJ. Prevalence of eosinophilic esophagitis in patients with refractory gastroesophageal reflux disease symptoms: a prospective study. Dig Liver Dis 2011; 43: 204-208.

32. Ates F, Yuksel ES, Higginbotham T, Slaughter JC, Mabary J, Kavitt RT, Garrett CG, Francis D, Vaezi MF. Mucosal impedance discriminates GERD from non-GERD conditions. Gastroenterology 2015; 148: 334-343.

33. Kline MM, Ewing M, Simpson N, Laine L. The utility of intraluminal impedance in patients with gastroesophageal reflux diseaselike symptoms but normal endoscopy and 24 hour $\mathrm{pH}$ testing. Clin Gastroenterology Hepatology 2008; 6: 880-889.

34. Patel A, Wang D, Sainani N, Sayuk GS, Gyawali CP. Distal mean nocturnal baseline impedance on $\mathrm{pH}$ impedance monitoring predicts reflux burden and symptomatic outcome in GERD. Aliment Pharmacol Ther 2016; 44: 890-898.

35. Frazzoni M, Savarino E, de Bortoli N, Martinucci I, Furnari M, Frazzoni L, Mirante VG, Bertani H, Marchi S, Conigliaro R, Savarino V. Analyses of the postreflux swallow-induced peristaltic wave index and nocturnal baseline impedance parameters increase the diagnostic yield of impedance-pH monitoring of patients with reflux disease. Clin Gastroenterol Hepatol 2016; 14: 40-46.
36. Taghavi SA, Ghasedi M, Saberi-Firoozi M, Alizadeh-Naeeni M, Bagheri-Lankarani K, Kaviani MJ, Hamidpour. Symptom association probability and symptom sensitivity index: preferable but still suboptimal predictors of response to high dose omeprazole. Gut 2005; 54: 1067-1071.

37. Slaughter JC, Goutte M, Rymer JA, Oranu AC, Schneider JA, Garrett CG, Hagaman D, Vaezi MF. Caution about overinterpretation of symptom indexes in reflux monitoring for refractory gastrooesophageal reflux disease. Clin Gastroenterol Hepatol 2011; 9: 868-874.

38. Kavitt RT, Higginbotham T, Slaughter JC, Patel D, Yuksel ES, Lominadze Z, Abou-Ismail A, Pasricha T, Garrett CG, Hagaman D, Vaezi MF. Symptom reports are not reliable during ambulatory monitoring. Am J Gastroenterol 2012; 107: 1826-1832.

39. Choksi Y, Slaughter JC, Sharda R, Higginbotham, Lal P, Vaezi MF. Symptom-associated probability does not reliably distinguish functional heartburn from reflux hypersensitivity. Aliment Pharmacol Ther 2018: 1-8.

40. Chae S, Richter J. Wireless 24, 48, and 96 hour or impedance or oropharyngeal prolonged $\mathrm{pH}$ monitoring: which test, when, and why for GERD? Current Gastroenterolgy Reports 2018; 20: 52 .

41. Ness-Jensen E, Hveem K, El-Serag H, Lagergren J. Lifestyle intervention in gastro-esophageal reflux disease. Clin Gastroenterol Hepatol 2106; 14 (2): 175-182.

42. Rodriguez-Stanley S, Ciociola AA, Zubaidi S, Proskin HM, Miner PB Jr. A single dose of ranitidine $150 \mathrm{mg}$ modulates oesophageal acid sensitivity in patients with functional heartburn. Aliment Pharmacol Ther 2004; 20: 975-982.

43. Broeders JA, Bredenoord AJ, Hazebroek EJ, Broeders IA, Gooszen HG, Smout AJ. Effects of anti-reflux surgery on weakly acidic reflux and belching. Gut 2011; 60: 435-441.

44. Bilgi MM, Vardar R, Yildirim E, Veznedaroglu B, Bor S. Prevalence of psychiatric comorbidity in symptomatic gastroesophageal reflux subgroups. Dig Dis Sci 2017; 62 (4): 984-993.

45. Fass R, Tougas G. Functional heartburn: The stimulus, the pain and the brain. Gut 2002; 51: 885-892.

46. Drossman D, Tack J, Ford A, Szighethy E, Tornblom H, Van Oudenhove L. Neuromodulators for functional gastrointestinal disorders (disorders of brain gut interaction): a Rome foundation working team work. Gastroenterology 2018; 154: 1140-1178.

47. Viazis N, Keyoglou A, Kanellopoulos AK, Karamanolis G, Vlachogiannakos J, Triantafyllou K, Ladas SD, Karamanolis DG. Selective serotonin reuptake inhibitors for the treatment of hypersensitive esophagus: a randomized, double-blind, placebo-controlled study. Am J Gastroenterol 2012; 107: 1662-1667.

48. Shapiro M, Shanani R, Taback H, Abramowich D, Scapa E, Broide E. Functional chest pain responds to biofeedback treatment, but functional heartburn does not; what is the difference? Eur J Gastroenterol Hepatol 2012; 24 (6): 708-714.

49. Richl ME, Pandolfino JE, Palsson OS, Keefer L. Feasibility and acceptability of esophageal-directed hypnotherapy for functional heartburn. Dis Esophagus 2016: 29 (5): 490-496.

50. Dickman R, Schiff E, Holland A, Wright C, Sarela SR, Han B, Fass R. Clinical trial: acupuncture vs. doubling the proton pump inhibitor dose in refractory heartburn. Aliment Pharmacol Ther 2017; 26 (10): 1333-1344. 